\title{
Plasma brain-derived neurotrophic factor daily variations in men: correlation with cortisol circadian rhythm
}

\author{
Silvia Begliuomini, Elena Lenzi, Filippo Ninni, Elena Casarosa, Sara Merlini, Nicola Pluchino, \\ Valeria Valentino, Stefano Luisi ${ }^{1}$, Michele Luisi and Andrea R Genazzani \\ Division of Gynecology and Obstetrics, Department of Reproductive Medicine and Child Development, University of Pisa, Pisa 56100, Italy \\ ${ }^{1}$ Division of Obstetrics and Gynecology, Department of Pediatric Obstetrics and Reproductive Medicine, University of Siena, Siena 53100, Italy \\ (Correspondence should be addressed to A R Genazzani; Email: a.genazzani@obgyn.med.unipi.it)
}

\begin{abstract}
Expression and secretion of neurotrophins, including brainderived neurotrophic factor (BDNF), are regulated also by neuronal activity. Data available in the literature suggest that BDNF central levels are influenced by light and dark. Diurnal changes of BDNF mRNA and protein contents have been demonstrated in the rat central nervous system. Based on these pieces of evidence, we investigated the hypothesis of a possible diurnal variation of BDNF circulating levels in human males. Moreover, we looked for a possible correlation with cortisol circadian rhythm, since both BDNF and cortisol are implicated in the maintenance of cerebral functions. In this study, 34 healthy young male volunteers were included. Five blood samples were drawn from each subject thrice in a month at regular 4-h intervals $(0800,1200,1600,2000$, and
\end{abstract}

$2400 \mathrm{~h}$ ). BDNF and cortisol were measured in all samples. BDNF was determined by ELISA method. Our results show that plasma BDNF levels, as well as cortisol levels, are significantly higher in the morning when compared with the night $(P<0 \cdot 001)$, with a trend of constant decrease during the day. Furthermore, plasma BDNF and cortisol are positively correlated (Spearman index $=0 \cdot 8466$ ). The present study is the first to demonstrate the presence of a diurnal rhythm of BDNF in humans. Moreover, the correlation found out between BDNF and cortisol circadian trend allows us to speculate that these two factors may be physiologically co-regulated, in order to maintain the homeostasis of integrated cerebral activities.

Journal of Endocrinology (2008) 197, 429-435

\section{Introduction}

Brain-derived neurotrophic factor (BDNF) is a member of the neurotrophin family expressed in many areas of the adult mammalian brain. Its biological action is mediated by the specific tyrosine kinase receptor trkB (Tapia Aranciba et al. 2004).

BDNF is recognized to play an important role in growth, differentiation, and survival of neurons during brain development (Bothwell 1995, Lewin \& Barde 1996), as well as in adulthood (Con Over \& Yancoupouolos 1997, Lu \& Figurov 1997). BDNF has also been shown to play an important role in activity-dependent synaptic plasticity in the hippocampus (Kang \& Schumann 1995, Korte et al. 1995), produce a lasting enhancement of synaptic efficacy in the dentate gyrus (Messaoudi et al.1998), and enhance glutamatergic synaptic transmission in hippocampus cell cultures through a presynaptic mechanism ( $\mathrm{Li}$ et al. 1998). It is possible that these effects may, in turn, enhance specific learning and memory processes and help to reduce cognitive deficits connected with aging and neurodegenerative disorders (Howells et al. 2000, Michalski \& Fahnestock 2003).

It is well established that neuronal activity regulates BDNF mRNA expression. Sensorial stimuli are able to influence
BDNF mRNA levels, as demonstrated by experiments based on light stimulation, in both developing and adult rats (Castrèn et al. 1992). Moreover, physical activity has been shown to increase BDNF mRNA in rat cerebral cortex and hippocampus (Neeper et al. 1991). Thus, the hippocampal BDNF expression largely depends on the neuronal excitation/inhibition balance (Castrèn et al. 1992), even if it appears to be also affected by corticosteroid hormones that seem to down-regulate it (Schaaf et al. 1998).

In recent years, many experimental studies in rats and mice indicate an endogenous cyclical change in central BDNF and trkB expressions within $24 \mathrm{~h}$ (Bova et al. 1998, Schaaf et al. 2000a, Dolci et al. 2003), although the implication of these circadian oscillations still remains unclear.

The suprachiasmatic nucleus ( $\mathrm{SCN}$ ) of the hypothalamus contains an endogenous oscillator that is the primary biological clock in mammals (Hastings 1997). Although the mechanisms underlying the endogenous clock rhythmicity are not yet fully characterized, recent findings suggest that BDNF may be involved in the light-regulated circadian pacemaker of the central nervous system (CNS; Liang et al. 2000).

First evidence for the presence of BDNF in human circulation emerged a decade ago (Rosenfeld et al. 1995). 
Since then many studies investigated the various sites of BDNF production in humans, in both neuronal and non-neuronal cells (Donovan et al. 1995, Yamamoto et al. 1996, Gielen et al. 2003). As there are no studies at present in the literature investigating a possible BDNF circadian rhythm in humans, we studied the BDNF levels throughout $24 \mathrm{~h}$ in healthy men, in order to detect the possible relative changes in plasma BDNF protein.

Additionally, we looked for a possible interplay between BDNF and cortisol, the circadian physiological rhythm of which is well recognized in human plasma, with a zenith in the morning and a large decrease during the day. For this purpose, we studied whether there are any similarities or divergences between plasma BDNF and cortisol physiological circadian behavior.

\section{Materials and Methods}

\section{Subjects}

Thirty-four young healthy male volunteers were recruited for this study. Their age was between 20 and 33 years (mean \pm S.D. $=25 \cdot 6 \pm 3 \cdot 2)$, with a body mass index (BMI) between $20 \cdot 5$ and $28 \cdot 3$ (mean \pm s.D. $=23 \cdot 9 \pm 1 \cdot 9$ ).

Prior to enrollment, participating subjects gave their written informed consent. The study was approved by the Ethics Committee of the Faculty of Medicine of the University of Pisa. Each subject was asked to answer a questionnaire regarding age, weight, height, chronic diseases, current illness, regular medication, allergies, or a family history of endocrinological, psychiatric, or neurological diseases. Physical examination and routine laboratory tests were performed and they disclosed no abnormalities. None of the subjects was taking psychoactive medications, hormone therapies, or anti-inflammatory drugs, and no mood or behavior disturbances were referred at the time of enrollment.

\section{Protocol}

In order to investigate circadian BDNF variations, blood from each subject was collected every $4 \mathrm{~h}$ for a total of five samples in $24 \mathrm{~h}$. At $0800 \mathrm{~h}$, after overnight fasting, the first blood sample was drawn from the cubital vein of each subject into EDTAcoated tubes (Vacutest Kima s.r.l., Arzergrande, Italy). Subsequently, blood sampling was repeated at 1200, 1600, 2000 , and $2400 \mathrm{~h}$. The tubes were kept on ice and, after collection, blood samples were immediately centrifuged at $4{ }^{\circ} \mathrm{C}$ (2500 $\boldsymbol{g}$ for $15 \mathrm{~min}$ ). Afterward, plasma was aliquoted and stored at $-80{ }^{\circ} \mathrm{C}$ until assay. For each subject, sampling was repeated thrice in a month in order to analyze possible intra-individual variations in BDNF and cortisol circadian changes.

\section{BDNF assay}

Plasma levels of BDNF were determined with an ELISA method (BDNF Emax Immunoassay System, Promega,
Madison, WI, USA), after appropriate dilution of samples (1:4) using block and Sample buffer, according to the manufacturer's instructions.

Briefly, 96-well flat-bottom immunoplates (Iwaki) were coated with anti-BDNF $\mathrm{mAb}$ and incubated at $4{ }^{\circ} \mathrm{C}$ overnight. After blocking by non-specific binding with block and sample buffer, standards and samples were added to the plates and incubated and shaken for $2 \mathrm{~h}$ at room temperature. Subsequently, after washing with TBST wash buffer, plates were incubated for $2 \mathrm{~h}$ with anti-human BDNF pAb. The last incubation required the addition of Anti-IgY-HRP conjugate. In the last step of the assay, TMB one solution was added in order to develop the colour. After stopping the reaction with $\mathrm{HCl}$ $1 \mathrm{M}$, the absorbance was read at $450 \mathrm{~nm}$ on a microplate reader and BDNF concentrations were determined automatically according to the BDNF standard curve (ranging from $7 \cdot 8$ to $500 \mathrm{pg} / \mathrm{ml}$ purified BDNF).

The entire procedure was performed using a semiautomated Basic Radim Immunoassay Operator (BRIORadim, Pomezia, Italy) equipped with a microplate reader of optical density. A computer system linked to the BRIO analyzed the final results and expressed them in $\mathrm{pg} / \mathrm{ml}$.

\section{Cortisol assay}

Plasma concentration of cortisol was determined by a specific commercially available RIA kit (Radim).

The sensitivity of the assay was $0.9 \mu \mathrm{g} / 1$. The intra- and inter-assay coefficients of variation were $2 \cdot 6$ and $8 \cdot 0 \%$ respectively.

\section{Parameters used and statistical analysis}

Plasma BDNF levels were expressed in $\mathrm{pg} / \mathrm{ml}$, whereas cortisol was expressed as $\mu \mathrm{g} / 1$. All data are reported as mean \pm s.D. Statistical analysis was carried out using GraphPad Prism 4.0 (San Diego, CA, USA). A Friedman test was performed, followed by a post hoc analysis with Dunn test. Percentages of decrease at each time point with respect to basal value (1200, 1600,2000 , and $2400 \mathrm{vs} 0800 \mathrm{~h}$ ) were calculated for both BDNF and cortisol. Finally, the correlation index (Spearman correlation coefficient) between BDNF and cortisol trends was calculated, based on the total 34 subjects $\times$ three samples $X$ five time points.

\section{Results}

Intra-individual variability in BDNF and cortisol measurements

We checked for possible intra-individual variations in BDNF and cortisol measurements by repeating blood samples thrice in a month for each subject (one blood sample every 10 days). The mean \pm s.D. of the three blood samples for each subject was calculated; subsequently, the means and standard deviations for each time point were computed, as reported in Tables 1 and 2 . 
Table 1 Intra-individual variability in brain-derived neurotrophic factor (BDNF) measurement: standard deviations of the means calculated for each subject $\times$ three blood samples at each time point

\begin{tabular}{|c|c|c|c|c|c|}
\hline & $0800 \mathrm{~h}$ & $1200 \mathrm{~h}$ & $1600 \mathrm{~h}$ & $2000 \mathrm{~h}$ & $2400 \mathrm{~h}$ \\
\hline Subject 1 & $8 \cdot 287792$ & $15 \cdot 86579$ & $23 \cdot 81197$ & $26 \cdot 12897$ & $5 \cdot 186842$ \\
\hline Subject 2 & $121 \cdot 2239$ & $22 \cdot 10611$ & $24 \cdot 59885$ & $7 \cdot 518643$ & $11 \cdot 42483$ \\
\hline Subject 3 & $15 \cdot 55635$ & $14 \cdot 13695$ & $11 \cdot 30133$ & $12 \cdot 9508$ & $6 \cdot 757465$ \\
\hline Subject 4 & $11 \cdot 37292$ & $8 \cdot 835723$ & $4 \cdot 68615$ & $6 \cdot 369458$ & $8 \cdot 778952$ \\
\hline Subject 5 & $11 \cdot 06662$ & $8 \cdot 538345$ & $14 \cdot 02581$ & $13 \cdot 78514$ & 7.989994 \\
\hline Subject 6 & $17 \cdot 89786$ & $29 \cdot 58485$ & $42 \cdot 42641$ & $12 \cdot 38669$ & 1·001665 \\
\hline Subject 7 & $23 \cdot 45854$ & $22 \cdot 18738$ & $12 \cdot 55083$ & $12 \cdot 30867$ & $10 \cdot 56456$ \\
\hline Subject 8 & $107 \cdot 3352$ & $19 \cdot 31942$ & $16 \cdot 59187$ & 4.978956 & $7 \cdot 18401$ \\
\hline Subject 9 & $134 \cdot 4917$ & $11 \cdot 45528$ & $17 \cdot 44735$ & $7 \cdot 60548$ & $10 \cdot 17988$ \\
\hline Subject 10 & $14 \cdot 40417$ & $12 \cdot 45913$ & 4.942671 & $18 \cdot 44641$ & $26 \cdot 45751$ \\
\hline Subject 11 & $3 \cdot 260368$ & $10 \cdot 59827$ & $6 \cdot 863672$ & $18 \cdot 40734$ & $5 \cdot 494543$ \\
\hline Subject 12 & $10 \cdot 98287$ & $9 \cdot 106225$ & $7 \cdot 218726$ & $12 \cdot 99654$ & $4 \cdot 513314$ \\
\hline Subject 13 & $9 \cdot 87269$ & $10 \cdot 61508$ & $7 \cdot 493998$ & $7 \cdot 076722$ & $5 \cdot 271622$ \\
\hline Subject 14 & $16 \cdot 78779$ & $6 \cdot 655073$ & $9 \cdot 696907$ & $14 \cdot 94925$ & $8 \cdot 425556$ \\
\hline Subject 15 & $11 \cdot 3377$ & $10 \cdot 91375$ & $13 \cdot 88488$ & $5 \cdot 896609$ & $24 \cdot 0211$ \\
\hline Subject 16 & $8 \cdot 861151$ & $18 \cdot 5262$ & $6 \cdot 409368$ & $9 \cdot 298925$ & $1 \cdot 868154$ \\
\hline Subject 17 & $28 \cdot 93095$ & $12 \cdot 00125$ & $9 \cdot 457272$ & $6 \cdot 413267$ & $6 \cdot 236185$ \\
\hline Subject 18 & $13 \cdot 2714$ & $5 \cdot 146844$ & $6 \cdot 819335$ & $4 \cdot 856954$ & $7 \cdot 672679$ \\
\hline Subject 19 & $7 \cdot 992496$ & $8 \cdot 006872$ & $13 \cdot 6504$ & $8 \cdot 43386$ & $10 \cdot 04092$ \\
\hline Subject 20 & $13 \cdot 99893$ & $2 \cdot 419366$ & $9 \cdot 551963$ & $6 \cdot 856384$ & $12 \cdot 25126$ \\
\hline Subject 21 & $7 \cdot 1631$ & $10 \cdot 92337$ & $6 \cdot 128621$ & $8 \cdot 764131$ & $5 \cdot 892368$ \\
\hline Subject 22 & $15 \cdot 12481$ & $2 \cdot 757716$ & $4 \cdot 259499$ & $12 \cdot 87478$ & $8 \cdot 608717$ \\
\hline Subject 23 & $15 \cdot 79335$ & $8 \cdot 016441$ & $4 \cdot 309292$ & $5 \cdot 38145$ & $4 \cdot 853864$ \\
\hline Subject 24 & $12 \cdot 75003$ & $2 \cdot 285461$ & $7 \cdot 979348$ & $5 \cdot 54617$ & $3 \cdot 459769$ \\
\hline Subject 25 & $6 \cdot 646804$ & $8 \cdot 822131$ & $14 \cdot 52859$ & $12 \cdot 6891$ & 1 \\
\hline Subject 26 & $8 \cdot 248636$ & $2 \cdot 357965$ & $2 \cdot 088061$ & $4 \cdot 562163$ & $9 \cdot 026627$ \\
\hline Subject 27 & $5 \cdot 444722$ & $4 \cdot 427565$ & $16 \cdot 1397$ & $11 \cdot 04596$ & $4 \cdot 2$ \\
\hline Subject 28 & $15 \cdot 27874$ & $7 \cdot 399324$ & $16 \cdot 34044$ & $6 \cdot 244998$ & $13 \cdot 25255$ \\
\hline Subject 29 & $23 \cdot 69578$ & $14 \cdot 26266$ & $4 \cdot 041452$ & $9 \cdot 455334$ & $3 \cdot 026549$ \\
\hline Subject 30 & 1.907878 & $4 \cdot 880915$ & $17 \cdot 55192$ & $3 \cdot 219213$ & $18 \cdot 21208$ \\
\hline Subject 31 & $10 \cdot 15332$ & $20 \cdot 71545$ & $11 \cdot 95031$ & $17 \cdot 22585$ & $13 \cdot 09313$ \\
\hline Subject 32 & $11 \cdot 88108$ & $10 \cdot 05037$ & $19 \cdot 20104$ & $10 \cdot 10149$ & 9.034932 \\
\hline Subject 33 & $10 \cdot 44031$ & $15 \cdot 06088$ & $12 \cdot 38184$ & $17 \cdot 38534$ & $9 \cdot 016282$ \\
\hline Subject 34 & $13 \cdot 31015$ & $6 \cdot 978777$ & $10 \cdot 43264$ & $14 \cdot 6186$ & 9.9985 \\
\hline Mean & $22 \cdot 00676811$ & $11 \cdot 1005$ & $12 \cdot 08125$ & $10 \cdot 49352$ & $8 \cdot 646953$ \\
\hline
\end{tabular}

\section{$B D N F$ circadian variations}

The highest BDNF level was found early in the morning $(827 \cdot 0 \pm 178 \cdot 3)$, with a progressive decrease during the day (Fig. 1). In particular, in the second blood sample, drawn at $1200 \mathrm{~h}$, plasma BDNF levels were significantly lower when compared with BDNF morning circulating levels $(P<0 \cdot 001)$. The BDNF levels further decreased during the day, so that values detected in the afternoon $(1600 \mathrm{~h})$ and the evening $(2000 \mathrm{~h})$ were significantly lower than those measured at $2400 \mathrm{~h}(P<0 \cdot 001)$. The lowest concentration was achieved at midnight $(214 \cdot 4 \pm 44 \cdot 3)(P<0.001$ vs $0800 \mathrm{~h}, P<0 \cdot 001$ vs 1600 h, $P<0 \cdot 001$ vs 2000 h).

Statistical results of the Friedman test and the difference in rank sum are reported in Table 3.

\section{Cortisol circadian variations}

We detected the highest plasma cortisol levels early in the morning $(226 \cdot 5 \pm 53 \cdot 1)$. Then, plasma cortisol showed a progressive decrease during the day, reaching a nadir at $1200 \mathrm{~h}$
$(61 \cdot 9 \pm 14 \cdot 0 ; P<0 \cdot 001$ vs 0800 h; Fig. 1b). In particular, 1600-h cortisol values were significantly lower than the morning values $(P<0.001$ vs $0800 \mathrm{~h})$. Moreover, cortisol plasma concentrations measured at $2000 \mathrm{~h}$ were significantly lower than those detected at $1200 \mathrm{~h}(P<0 \cdot 001)$ and, analogously, $2400 \mathrm{~h}$ cortisol levels were significantly lower than those measured at $1600 \mathrm{~h}(P<0 \cdot 001)$.

Statistical results of the Friedman test and the difference in rank sum are reported in Table 4.

\section{BDNF/cortisol correlation}

In order to strengthen the observation of a similarity in BDNF and cortisol decreasing trend, we calculated the percentage of decrease in both BDNF and cortisol with respect to the maximum value detected in the morning. The correspondence between BDNF and cortisol circadian decreasing trend was attested by the determination of the Spearman index $(0 \cdot 8466)$ that was calculated based on the total 34 subjects $X$ three times a month $X$ five tests per day. 
Table 2 Intra-individual variability in cortisol measurement: standard deviations of the means calculated for each subject $\times$ three blood samples at each time point

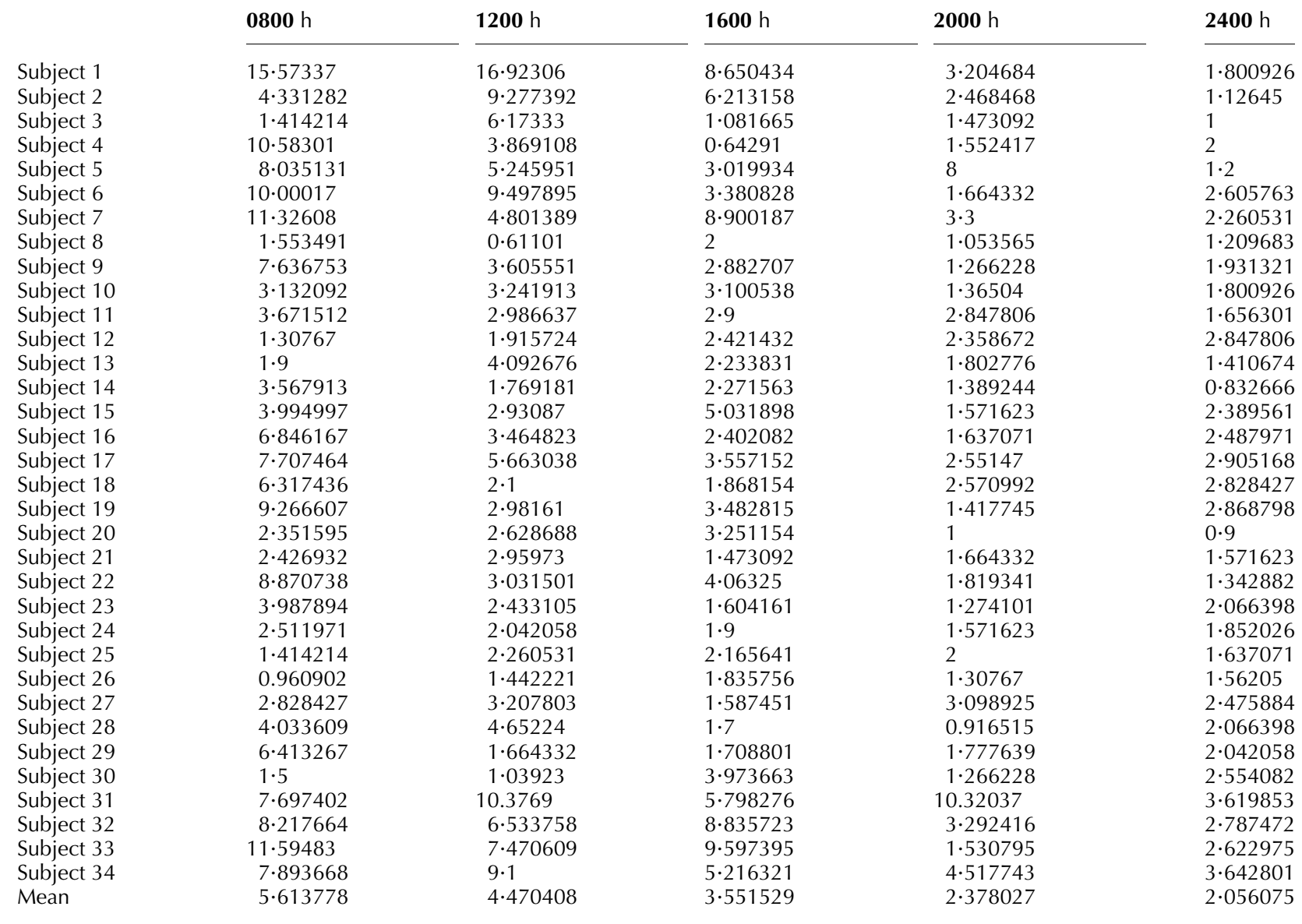

\section{Discussion}

The primary purpose of the present study was to investigate the presence of a possible circadian rhythm in BDNF circulating levels in humans.

In our previous study, we found that BDNF circulating levels are closely related to the sex hormonal status, pointing out the key role played by sex gonadal hormones in the modulation of expression and production of BDNF (Begliuomini et al. 2007). However, it is reasonable to suppose that changes in the plasma BDNF levels are not only endocrine based, but also influenced by other factors, such as neurotransmitters, sensorial stimuli, and physical activity.

Even though no studies have been yet published about circadian oscillations of BDNF in humans, many pieces of experimental evidence in rats corroborate the hypothesis of a circadian BDNF rhythm. Light:darkness cycles could in some way influence BDNF expression by modulating the cerebral circadian pacemaker localized in the SCN of the hypothalamus. It has been shown that both BDNF mRNA and protein, as well as trkB receptor, present rhythmic variations in rat CNS (Bova et al. 1998, Berchtold et al. 1999). Bova et al. (1998) and Berchtold et al. (1999) analyzed the expression of BDNF mRNA in the rat hippocampus and frontal cortex and they both observed that the highest BDNF mRNA levels were reached during the dark hours (activity period), while the lowest BDNF levels were detected during the light hours (rest period).

The present results show that plasma BDNF in human males presents a characteristic trend during the day; in our study population, the highest BDNF concentrations were detected in the morning, followed by a substantial decrease throughout the day and the lowest values were observed at midnight. Evidently, this decline in BDNF during the day may be ascribed to a circadian secretory model. In fact, it has been shown that BDNF presents a very short half-life in plasma $\left(t_{1 / 2}=0.92 \mathrm{~min}\right.$; Poduslo \& Curran 1996), and then it is conceivable that BDNF is secreted with a pulsatory circadian rhythm, featured by a progressive reduction in the amplitude of the pulses throughout the day.

Furthermore, our results point out that the daily trend of plasma BDNF is very similar to the trend of cortisol. Cortisol 


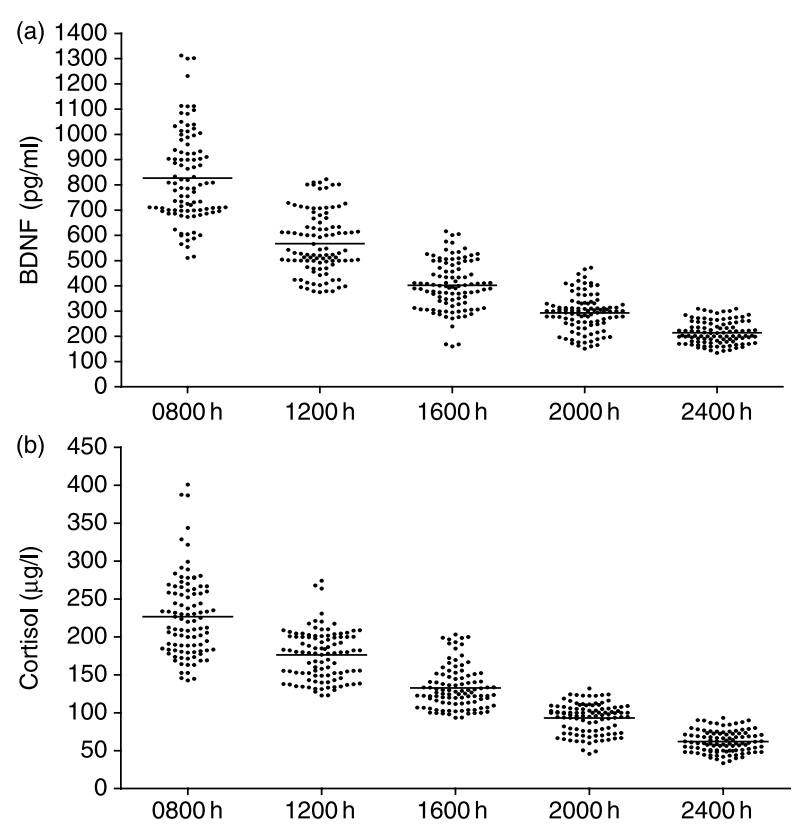

Figure 1 Scatter plot representing the decreasing trend of (a) plasma BDNF $(\mathrm{pg} / \mathrm{ml})$ and (b) cortisol $(\mu \mathrm{g} / \mathrm{l})$ throughout the day in healthy males.

is the most important glucocorticoid in humans and, besides its well-known effects on metabolism, bone, and blood pressure, it has been recognized to play a key role in the homeostasis of the CNS. The pulsatile secretion of cortisol in humans has been well established; its pulses have frequencies in the range of 60-90 min. Regulation of cortisol secretion depends on the integrity of the hypothalamic-pituitaryadrenal axis. In physiological conditions, hypothalamic CRF stimulates the pituitary to produce adrenocorticotrophin (ACTH) that, in turn, has a stimulatory effect on cortisol production by the adrenal cortex. Cortisol exerts a negative feedback with a long-loop mechanism, so that high circulating cortisol levels are associated with low CRF levels

Table 3 Results of post hoc analysis of brain-derived neurotrophic factor (BDNF) circadian rhythm by the means of Dunn's test

\begin{tabular}{|c|c|c|}
\hline & Difference in rank sum & $P$ value \\
\hline \multicolumn{3}{|l|}{ Dunn's test } \\
\hline 0800 vs $1200 \mathrm{~h}$ & $88 \cdot 00$ & $<0 \cdot 001$ \\
\hline 0800 vs $1600 \mathrm{~h}$ & $189 \cdot 0$ & $<0 \cdot 001$ \\
\hline 0800 vs $2000 \mathrm{~h}$ & $285 \cdot 0$ & $<0 \cdot 001$ \\
\hline 0800 vs $2400 \mathrm{~h}$ & $378 \cdot 0$ & $<0 \cdot 001$ \\
\hline 1200 vs $1600 \mathrm{~h}$ & $101 \cdot 0$ & $<0 \cdot 001$ \\
\hline 1200 vs $2000 \mathrm{~h}$ & $197 \cdot 0$ & $<0 \cdot 001$ \\
\hline 1200 vs $2400 \mathrm{~h}$ & $290 \cdot 0$ & $<0 \cdot 001$ \\
\hline 1600 vs $2000 \mathrm{~h}$ & $96 \cdot 00$ & $<0 \cdot 001$ \\
\hline 1600 vs $2400 \mathrm{~h}$ & $189 \cdot 0$ & $<0 \cdot 001$ \\
\hline 2000 vs $2400 \mathrm{~h}$ & $93 \cdot 00$ & $<0 \cdot 001$ \\
\hline
\end{tabular}

Value of Friedman test is $370.8(P<0.0001)$.
Table 4 Results of post hoc analysis of cortisol circadian rhythm by the means of Dunn's test

\begin{tabular}{|c|c|}
\hline Difference in rank sum & $P$ value \\
\hline $61 \cdot 5$ & $>0.05(\mathrm{NS})$ \\
\hline $169 \cdot 5$ & $<0 \cdot 001$ \\
\hline $271 \cdot 0$ & $<0 \cdot 001$ \\
\hline $368 \cdot 0$ & $<0 \cdot 001$ \\
\hline $108 \cdot 0$ & $<0 \cdot 001$ \\
\hline $209 \cdot 5$ & $<0.001$ \\
\hline $306 \cdot 5$ & $<0.001$ \\
\hline $101 \cdot 5$ & $<0 \cdot 001$ \\
\hline $198 \cdot 5$ & $<0 \cdot 001$ \\
\hline $97 \cdot 0$ & $<0.001$ \\
\hline
\end{tabular}

Value of Friedman test is $371.3(P<0.0001)$.

and vice versa (Young et al. 2004). Disruption in cortisol rhythm has been observed in many pathological conditions, such as major depression, sleep, and mood disorders, as well as in acute or chronic psychophysical stress conditions (Chrousos \& Gold 1992, Erickson et al. 2003).

Since a down-regulation by corticosteroid hormones on BDNF mRNA and protein in the rat has been reported (Schaaf et al. 1998, 2000b), we verified whether there was a relationship in humans between cortisol and BDNF plasma levels in physiological conditions.

The present results underline a positive correlation between plasma BDNF and cortisol daily trend, thus suggesting a possible co-regulation of the expression of these two compounds. Additionally, the similarity in the circadian variations of BDNF and cortisol allows us to hypothesize that glucocorticoid and neurotrophic tone may have a synergic role in the homeostasis of cerebral functions.

In pre-clinical studies, it has been shown that endogenous or exogenous corticosterone, which represents the main glucocorticoid in the rat, suppresses central BDNF mRNA and peptide expressions (Schaaf et al. 1998). This might be related to the inhibition of BDNF synthesis mediated by the activation of mineralocorticoid and glucocorticoid receptors (Schaaf et al. 2000b). On the other hand, the role of corticosterone in regulating the BDNF levels is still a subject of debate. In fact, it has been shown in the animal model that stress can decrease hippocampal BDNF, also in adrenalectomized rats, suggesting that corticosterone, $\mathrm{ACTH}$, and CRF are not the only elements of stress response contributing to the observed decrease in the BDNF levels in rats (Smith et al. 1995).

Our results, demonstrating a consensual diminishing trend of BDNF and cortisol plasma levels in a physiological 24-h cycle, seem to enter in conflict with studies on the rat hippocampus. Our data suggest a fairly consistent co-regulation of plasma BDNF and cortisol, mediated by an unknown mechanism, rather than a down-regulation of BDNF by corticosteroids. 
Alternatively, the hypothesis of a down-regulation of BDNF by corticosteroids could be sustained if we consider the delay between the BDNF mRNA expression and the subsequent process of translation, synthesis, and release of BDNF plasma protein. From this point of view, it would be reasonable to suppose that high levels of cortisol may inhibit the BDNF mRNA expression in the CNS, but this phenomenon cannot be immediately observed at the peripheral level.

However, it has recently been demonstrated that chronically enhanced cortisol induces an augmentation in BDNF in primates (McMillan et al. 2004), thus constituting a compensatory mechanism in response to cortisol-induced neurotoxicity. These discrepancies may also be influenced by a differential distribution of the glucocorticoid and mineralocorticoid receptors in rats and humans (Sanchez et al. 2000).

In conclusion, our results demonstrate that, in physiological conditions, circulating BDNF in humans presents a characteristic daily variation that is closely similar to the cortisol circadian rhythm. In our opinion, this may be explained by the presence of an individual internal balance that assures a homeostasis between protective and insulting factors at the neuronal level. The correct functioning of this compensatory mechanism may be responsible for maintaining appropriate levels of activity in the hippocampus and other brain areas.

\section{Acknowledgements}

This work was partially supported by a grant from the Fondazione Cassa Risparmio di San Miniato, San Miniato, Italy. The authors would also like to thank Dr Massimetti for statistical advice and Dr Monteleone for linguistic revision. The authors declare that there is no conflict of interest that would prejudice the impartiality of this scientific work.

\section{References}

Begliuomini S, Casarosa E, Pluchino N, Lenzi E, Centofanti M, Freschi L, Pieri M, Genazzani AD, Luisi S \& Genazzani AR 2007 Influence of endogenous and exogenous sex hormones on plasma brain-derived neurotrophic factor. Human Reproduction 22 995-1002.

Berchtold NC, Oliff HS, Isaksson P \& Cotman CW 1999 Hippocampal BDNF mRNA shows a diurnal regulation, primarily in the exon III transcript. Molecular Brain Research 71 11-22.

Bothwell M 1995 Functional interactions of neurotrophin receptors. Annual Reviews of Neuroscience 18 223-253.

Bova R, Micheli MR, Quadralucci P \& Zucconi GG 1998 BDNF and trkB mRNA oscillate in the rat brain during the light-dark cycle. Molecular Brain Research 57 321-324.

Castrèn E, Zafra H, Thoenen H \& Lindholn D 1991 Interplay between glutamate and gamma-aminobutirric acid transmitter systems in the physiological regulation of brain-derived neurotrophic factor and nerve growth factor synthesis in hippocampal neurons. PNAS 88 10037-10041.

Castrèn E, Zafra H, Thoenen H \& Lindholn D 1992 Light regulates expression of brain-derived neurotrophic factor mRNA in rat visual cortex. PNAS 89 9444-9448.
Chrousos GP \& Gold PW 1992 The concepts of stress and stress system disorders. Overview of physical and behavioral homeostasis. Journal of the American Medical Association 267 1244-1252.

Con Over JC \& Yancoupoulos GD 1997 Neurotrophin regulation of the developing nervous system: analyses of knockout mice. Reviews in Neuroscience 8 13-27.

Dolci C, Montaruli A, Roveda E, Barajon A, Vizzotto GG, Zucconi GG \& Caradente F 2003 Circadian variations in expression of the trkB receptor in adult rat hippocampus. Brain Research 994 67-72.

Donovan MJ, Miranda RC, Kraemer R, McCaffrey TA, Tessarollo L, Mahadeo D, Sharif S, Kaplan DR, Tsoulfas P \& Parada L 1995 Neurotrophin and neurotrophin receptors in vascular smooth muscle cells. Regulation of expression in response to injury. American Journal of Pathology 147 309-324.

Erickson K, Drevets W \& Schulkin J 2003 Glucocorticoid regulation of diverse cognitive functions in normal and pathological emotional states. Neuroscience Biobehaviour Review 27 233-246.

Gielen A, Khademi M, Muhallab S, Olsson T \& Piehl F 2003 Increased brainderived neurotrophic factor expression in white blood cells of relapsingremitting multiple sclerosis patients. Scandinavian Journal of Immunology $\mathbf{5 7}$ 493-497.

Hastings MH 1997 Central clocking. Trends in Neuroscience $20459-464$.

Howells DW, Porritt MJ, Wong JY, Batchelor PE, Kalnins R, Hughes AJ \& Donnan GA 2000 Reduced BDNF mRNA expression in the Parkinson's disease substantia nigra. Experimental Neurology 166 127-135.

Kang H \& Schumann EM 1995 Long-lasting neurotrophin-induced enhancement of synaptic transmission in the adult hippocampus. Science $\mathbf{2 6 7}$ $1658-1662$.

Korte M, Carroll P, Wolf E, Brem G, Thoenen H \& Bonhoeffer T 1995 Hippocampal long-term potentiation is impaired in mice lacking brainderived neurotrophic factor. PNAS 92 8856-8869.

Lewin GR \& Barde YA 1996 Physiology of the neurotrophins. Annual Reviews of Neuroscience 19 289-317.

Li YX, Zhang Y, Lester HA, Schumann E \& Davidson N 1998 Enhancement of neurotransmitter release induced by brain-derived neurotrophic factor in cultured hippocampal neurons. Journal of Neuroscience 18 10231-10240.

Liang FQ, Allen G \& Earnest D 2000 Role of brain-derived neurotrophic factor in the circadian regulation of the suprachiasmatic pacemaker by light. Journal of Neuroscience 20 2978-3978.

Lu B \& Figurov A 1997 Role of neurotrophins in synapse development and plasticity. Reviews in Neuroscience 8 1-12.

McMillan PJ, Wilkinson CW, Greenup L, Raskind MA, Peskind ER \& Leverenz JB 2004 Chronic cortisol exposure promotes the development of a GABAergic phenotype in the primate hippocampus. Journal of Neurochemistry 91 843-851.

Messaoudi E, Barsden K, Srebro B \& Bramham CR 1998 Acute intrahippocampal infusion of BDNF induces lasting potentiation of synaptic transmission in the rat dentate gyrus. Journal of Neurophysiology 79 496-499.

Michalski B \& Fahnestock M 2003 Pro-brain-derived neurotrophic factor is decreased in parietal cortex in Alzheimer's disease. Molecular Brain Research 111 148-154.

Neeper SA, Gonez-Pinhilla F, Choi J \& Cotman CW 1991 Physical activity increases mRNA for brain-derived neurotrophic factor and nerve growth factor in rat brain. Molecular and Cellular Biology 11 143-153.

Poduslo JF \& Curran GL 1996 Permeability at the blood-brain and bloodnerve barriers of the neurotrophic factors: NGF, CNTF, NT-3, BDNF. Molecular Brain Research 36 280-286.

Rosenfeld RD, Zeni L, Haniu M, Talvenheimo J, Radka SF, Bennett L, Miller JA \& Welcher AA 1995 Purification and identification of brain-derived neurotrophic factor from human serum. Protein Expression and Purification 6 465-471.

Sanchez MM, Young LJ, Plotsky PM \& Insel TR 2000 Distribution of corticosteroid receptors in the rhesus brain: relative absence of glucocorticoid receptors in the hippocampal formation. Journal of Neuroscience 20 4657-4668. 
Schaaf MJM, Durland R, de Kloet ER \& Vreugdenhil E 1998 Downregulation of BDNF $\mathrm{mRNA}$ and protein in rat hippocampus by corticosterone. Brain Research 813 112-120.

Schaaf MJM, Durland R, de Kloet ER \& Vreugdenhil E 2000a Circadian variation in BDNF mRNA in the rat hippocampus. Molecular Brain Research $75342-344$

Schaaf MJM, De Kloet ER \& Vreugdenhil E $2000 b$ Corticosterone effects on BDNF expression in the hippocampus. Implications for memory formation. Stress 3 201-208.

Smith MA, Makino S, Kvetnansky R \& Post RM 1995 Stress and glucocorticoids affect the expression of brain-derived neurotrophic factor and neurotrophin-3 mRNAs in the hippocampus. Journal of Neuroscience 15 1768-1777.

Tapia-Arancibia L, Rage F, Givalois L \& Arancibia S 2004 Phisiology of BDNF: focus on hypothalamic function. Frontiers in Neuroendocrinology 25 77-107.
Yamamoto M, Sobue G, Yamamoto K, Terao S \& Mitsuma T 1996 Expression of mRNAs neurotrophic factors (NGF, BDNF, NT-3 and GDNF) and their receptors (p75NGFR, TrkA, TrkB,and Trkc) in the adult human peripheral nervous system and non-neural tissues. Neurochemistry Research $\mathbf{4 1 9}$ 929-938.

Young EA, Abelson J \& Lightman SL 2004 Cortisol pulsatility and its role in stress regulation and health. Frontiers in Neuroendocrinology 25 69-76.

Received in final form 30 January 2008

Accepted 15 January 2008

Made available online as an Accepted Preprint 15 January 2008 